\title{
MAGNETOELASTIC RESONANCE IN A CRYSTAL WITH LATTICE PHASE TRANSITION
}

\author{
V.G. BARYAKHTAR, A.G. DANILEVICH, V.A. L'VOV
}

PACS 05.70.Fh, 62.20.-x,

The possibility of the abnormally strong repulsion of the quasisound and quasispin branches of the wave spectrum in a cubic ferromagnetic crystal due to the phase transition in a lattice is predicted.

\section{Introduction}

The coupled magnetoelastic waves in ferromagnetic and antiferromagnetic crystals are widely studied during a long time (see [1-5] and references therein). The magnetoelastic waves in the crystals undergoing the spinreorientation transitions attracted a special interest because of the strong effect of magnetoelastic coupling on the wave spectrum. The effects of magnetoelastic coupling are the most pronounced in a vicinity of the phase transition points $[5,6]$, because the spin-reorientation transitions are accompanied by a decrease of the energy gap in the spin-wave spectrum. When the energy gap becomes comparable in value with the value of "repulsion" of the quasispin and quasisound branches of the spectrum, the magnetoelastic coupling leads to a substantial reduction of the velocity of a quasisound magnetoelastic wave. The theory predicts the possibility of vanishing the sound velocity, i.e., the conversion of the linear dispersion law of the quasisound into the quadratic one $[5$, $6,7]$.

Ferromagnetic shape memory alloys (FSMAs) are considered now as a new class of materials which are of interest in both applied and academic aspects [8-10]. The specific properties of these alloys are caused by their martensitic transformation (MT) which is accompanied by a spontaneous deformation of the crystal lattice and a pronounced softening of the shear modulus. In particular, the Ni-Mn-Ga alloys, which exhibit the cubictetragonal MT, are intensively studied. The most intriguing feature of these materials is a giant (> 5\%) magnetically induced deformation, which is caused by the transformation of the twin structure of a single crystalline alloy specimen under the external magnetic field.
The ordinary magnetostriction of the $\mathrm{Ni}-\mathrm{Mn}-\mathrm{Ga}$ alloys has been studied in both experimental $[11,12]$ and theoretical ways [13]. The drastic increase of the magnetostriction was observed, while the temperature of a cubic crystal approaches the MT temperature [10].

To the best of our knowledge, the coupled magnetoelastic waves in the FSMAs were not considered jet. The experimentally observed increase of the magnetostriction and a softening of the shear elastic modulus suggest an idea of the strong effect of magnetoelastic coupling on the wave spectrum. In the present article, the theory of magnetoelastic coupling is applied to a single crystal, whose temperature approaches the cubic-tetragonal phase transition temperature. The general theoretical relationships are used for the quantitative description of the spectrum of coupled magnetoelastic waves.

\section{Formalism}

To describe the coupled magnetoelastic waves, the free energy of a $\mathrm{Ni}-\mathrm{Mn}-\mathrm{Ga}$ single crystal should be presented as

$F=F_{m}+F_{e}+F_{m e}$.

The first term is the energy of the magnetic subsystem of the cubic crystal

$F_{m}=\frac{\alpha}{2} \frac{\partial \mathbf{M}}{\partial x_{i}} \frac{\partial \mathbf{M}}{\partial x_{k}}+\frac{K_{1}}{M^{4}}\left(M_{x}^{2} M_{y}^{2}+M_{x}^{2} M_{z}^{2}+M_{y}^{2} M_{z}^{2}\right)+$

$+\frac{K_{2}}{M^{6}} M_{x}^{2} M_{y}^{2} M_{z}^{2}-\mathbf{M H}$

where $\mathbf{M}$ and $\mathbf{H}$ are the magnetization and magnetic field vectors, $\alpha_{i k}$ is the exchange energy constant, and $K_{1}$ and $K_{2}$ are the magnetocrystalline anisotropy constants, respectively. The second term,

$$
\begin{aligned}
& F_{e}=\frac{3}{2}\left(C_{11}+2 C_{12}\right) u_{1}^{2}+\frac{1}{6} C^{\prime}\left(u_{2}^{2}+u_{3}^{2}\right)+ \\
& +2 C_{44}\left(u_{4}^{2}+u_{5}^{2}+u_{6}^{2}\right)
\end{aligned}
$$


is the elastic energy expressed in terms of linear combinations of the strain tensor components $u_{1}=\left(\varepsilon_{x x}+\right.$ $\left.\varepsilon_{y y}+\varepsilon_{z z}\right) / 3, u_{2}=\sqrt{3}\left(\varepsilon_{x x}-\varepsilon_{y y}\right), u_{3}=2 \varepsilon_{z z}-\varepsilon_{x x}-\varepsilon_{y y}$, $u_{4}=\varepsilon_{y z}, u_{5}=\varepsilon_{x z}$, and $u_{6}=\varepsilon_{x y}$ and the elastic moduli of a cubic crystal $C_{11}, C_{12}, C_{44}$, and $C^{\prime}=\left(C_{11}-C_{12}\right) / 2$. The third term,

$F_{m e}=-\frac{\delta_{2}}{M^{2}}\left\{\sqrt{3} u_{2}\left(M_{x}^{2}-M_{y}^{2}\right)+\right.$

$\left.+u_{3}\left(2 M_{z}^{2}-M_{x}^{2}-M_{y}^{2},\right)\right\}$

is an essential part of the magnetoelastic coupling energy, which causes the interaction of spin waves with the soft phonon mode (second sound) [10]. The coordinate frame is related to $\langle 100\rangle$ crystallographic directions of the cubic phase.

Now, the magnetization $\mathbf{M}$ is presented as the sum of its equilibrium part $\mathbf{M}_{0}$ and the small additive $\mathbf{m}$ that satisfies the equation

$\dot{\mathbf{m}}=-\gamma \mathbf{M}_{0} \times \mathbf{H}_{\mathrm{eff}}$,

where $\gamma$ is the gyromagnetic ratio, and $\mathbf{H}_{\text {eff }}=-\delta F / \delta \mathbf{m}$ is an effective magnetic field, which acts on the magnetization vector in the nonequilibrium magnetic state. To complete the system of equations for magnetoelastic waves, the equation for displacements of atoms, $\mathbf{U}=\mathbf{U}_{0} \exp \{i(\mathbf{k r}-\omega t)\}$, in the crystal should be used. (Here, the symbols $\mathbf{k}$ and $\omega$ are the wave vector and the angular frequency, respectively). The energies given by Eqs. (3) and (4) can be expressed through the displacements using the commonly known relation $2 \varepsilon_{l m}=$ $\left(\partial U_{l} / \partial x_{m}\right)+\left(\partial U_{m} / \partial x_{l}\right)$. The dynamic equation for displacements is

$\rho \ddot{\mathbf{U}}=-\frac{\delta F}{\delta \mathbf{U}}$.

The pure second sound has the frequency $\omega_{p h}=$ $\left(C^{\prime} / \rho\right)^{1 / 2} k$ and the wave vector $\mathbf{k} \|\langle 110\rangle$. For the sake of definiteness, let the wave vector be parallel to [110], and let the vector $\mathbf{M}_{0}$ be oriented in the $X O Z$ plane. In this case, we have

$$
\left(\begin{array}{l}
M_{0 x} \\
M_{0 y} \\
M_{0 z}
\end{array}\right)=\left(\begin{array}{c}
M_{0} \sin \theta \\
0 \\
M_{0} \cos \theta
\end{array}\right),
$$

where $\theta$ is the angle between the vector $\mathbf{M}_{0}$ and the $z$-axis. By presenting the energy (Eq. (1)) as a series in the components of the vectors $\mathbf{U}$ and $\mathbf{m}=$ $\mathbf{m}_{0} \exp \{i(\mathbf{k r}-\omega t)\}$, one can obtain the equation that describes the spatial dispersion of the coupled magnetoelastic waves. This equation has a form

$\left(\omega^{2}-s_{t 1}^{2} k^{2}\right)\left\{\left(\omega^{2}-s_{t 2}^{2} k^{2}\right)\left[\omega^{2}-s_{l 2}^{2} k^{2}\right] \times\right.$

$\times\left[\omega^{2}-\gamma^{2}\left(M_{0 z}^{2} \omega_{1} \omega_{2}+M_{0 x}^{2} \omega_{3} \omega_{4}\right)\right]-$

$-72 \delta_{2}^{2} \gamma^{2} k^{2} M_{0 x}^{2} M_{0 z}^{2}\left[\omega^{2}-\left(s_{l 1}^{2}+s_{t 1}^{2}\right) k^{2} / 2\right] \times$

$\left.\times\left(M_{0 z}^{2} \omega_{1}+M_{0 x}^{2} \omega_{4}\right) / \rho M_{0}^{6}\right\}=0$,

where

$s_{l 1}^{2}=C_{11} / \rho, s_{l 2}^{2}=\left(C_{11}+C_{12}+2 C_{44}\right) / 2 \rho, s_{t 1}^{2}=C_{44} / \rho$,

$s_{t 2}^{2}=\left(C_{11}-C_{12}\right) / 2 \rho=C^{\prime} / \rho$,

$\omega_{1}=\alpha k^{2}+H_{z} / M_{0 z}+2 K_{1} M_{0 z}^{2} / M_{0}^{4}+2 K_{2} M_{0 x}^{2} M_{0 z}^{2} / M_{0}^{6}$,

$\omega_{2}=\alpha k^{2}+H_{z} / M_{0 z}+2 K_{1}\left(M_{0 x}^{4}-6 M_{0 x}^{2} M_{0 z}^{2}+M_{0 z}^{4}\right) / M_{0}^{6}$,

$\omega_{3}=\alpha k^{2}+H_{x} / M_{0 x}+2 K_{1}\left(M_{0 x}^{4}-6 M_{0 x}^{2} M_{0 z}^{2}+M_{0 z}^{4}\right) / M_{0}^{6}$,

$\omega_{4}=\alpha k^{2}+H_{x} / M_{0 x}+2 K_{1} M_{0 x}^{2} / M_{0}^{4}+2 K_{2} M_{0 x}^{2} M_{0 z}^{2} / M_{0}^{6}$.

When the magnetoelastic parameter $\delta_{2}$ is equal to zero, Eq. (8) has the solutions $\omega_{l}^{(1)}=s_{l 1} k, \omega_{l}^{(2)}=s_{l 2} k$, $\omega_{t}^{(1)}=s_{t 1} k, \omega_{t}^{(2)}=s_{t 2} k \equiv \omega_{p h}$ and $\omega_{s w}=\gamma\left(M_{0 z}^{2} \omega_{1} \omega_{2}+\right.$ $\left.M_{0 x}^{2} \omega_{3} \omega_{4}\right)^{1 / 2}$, which correspond to the uncoupled elastic and spin waves, respectively. The solutions describing the coupled magnetoelastic waves are studied below.

\section{Results}

The magnetoelastic term in Eq. (8) includes the factor $\delta_{2}^{2} M_{0 x}^{2} M_{0 z}^{2} \propto \sin ^{2} \theta \cos ^{2} \theta$, and therefore, the interaction between the spin waves and the soft elastic mode vanishes when $\theta=0, \pi / 2$ and reaches the maximal value when $\theta=\pi / 4$, i.e., when the magnetization vector is aligned with [101] crystallographic direction. Under the usual experimental conditions, an external magnetic field is strong enough to align the magnetization vector along the field direction. As so, the case where the magnetic field is parallel to [101] crystallographic direction is especially interesting. In this case, Eq. (8) is simplified to the form

$\left(\omega^{2}-s_{t 1}^{2} k^{2}\right)\left\{\left(\omega^{2}-s_{t 2}^{2} k^{2}\right) \times\right.$ 

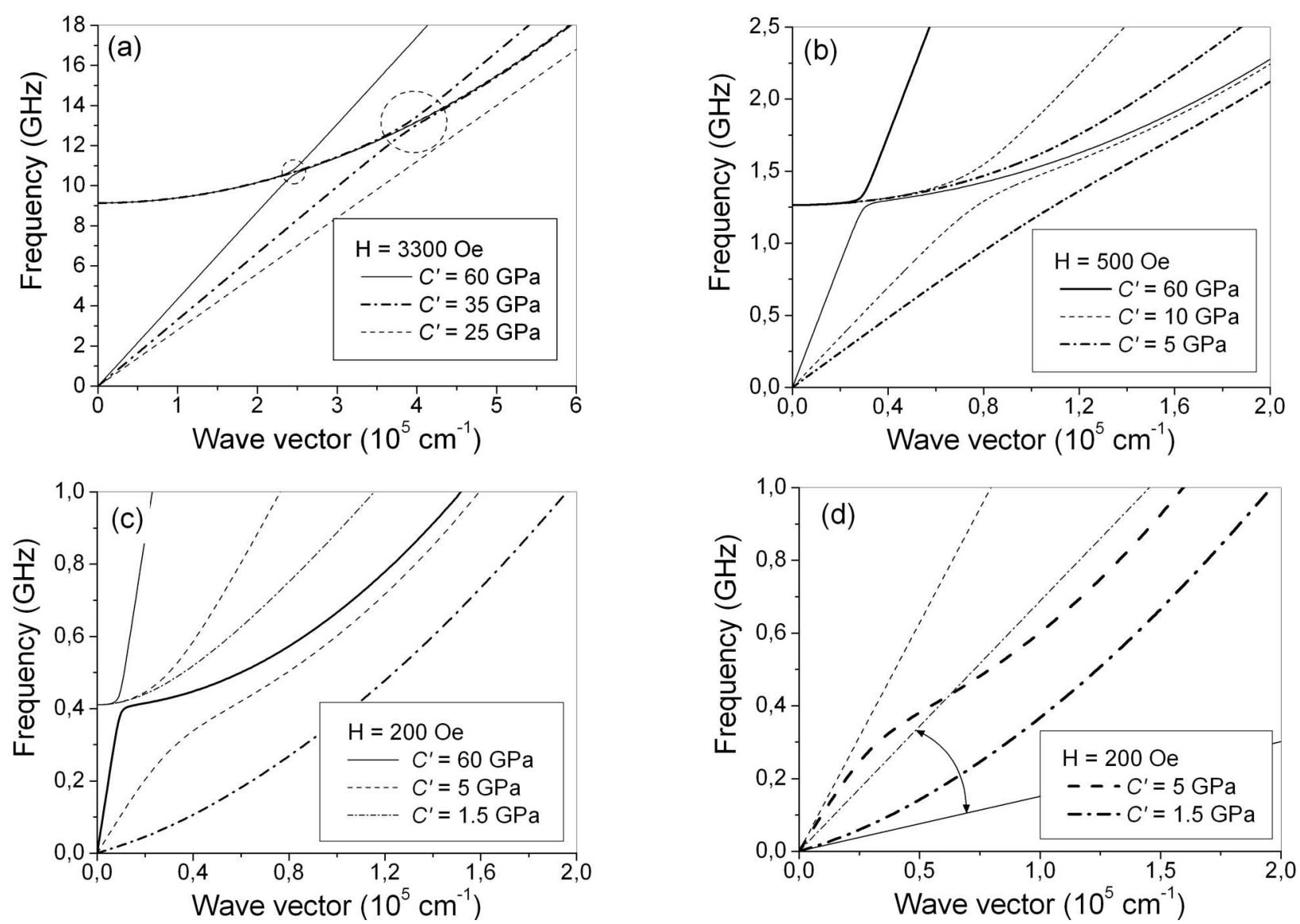

Dispersion of coupled magnetoelastic waves: the dispersion curves presented in $(a),(b)$, $(c)$ were computed for various values of the shear elastic modulus and the external magnetic field $(\mathbf{H} \|[101])$; the initial fragments of the dispersion curves presented in ( $d$ ) were computed in the cases where $\mathbf{H} \|$ [100] (thin lines) and $\mathbf{H} \|$ [101] (bold lines). A slope of the thin solid line corresponds to the sound velocity reduced by the magnetoelastic interaction

$\times\left(\omega^{2}-s_{l 2}^{2} k^{2}\right)\left(\omega^{2}-\gamma^{2} M_{0}^{2} \omega_{m 1} \omega_{m 2}\right)-$

$\left.-18 \delta_{2}^{2} \gamma^{2} k^{2}\left[\omega^{2}-\left(s_{l 1}^{2}+s_{t 1}^{2}\right) k^{2} / 2\right] \omega_{m 1} / \rho\right\}=0$,

where

$\omega_{m 1}=\alpha k^{2}+H / M_{0}+K_{1} / M_{0}^{2}+K_{2} / 2 M_{0}^{2}$,

$\omega_{m 2}=\alpha k^{2}+H / M_{0}-2 K_{1} / M_{0}^{2}$.

According to the available experimental data, the equilibrium direction of the magnetization vector of a $\mathrm{Ni}-$ $\mathrm{Mn}-\mathrm{Ga}$ single crystal is parallel to $\langle 100\rangle$, and the values of magnetic anisotropy constants measured at $T=295$ $\mathrm{K}$ are $K_{1}=2.7 \times 10^{4} \mathrm{erg} / \mathrm{cm}^{3}, K_{2}=-6.1 \times 10^{4} \mathrm{erg} / \mathrm{cm}^{3}$; the reported value of saturation magnetization is close to $600 \mathrm{G}$ [14].

The values $\rho \approx 8 \mathrm{~g} / \mathrm{cm}^{3}$ and $\delta_{1} \approx 10^{7} \mathrm{erg} / \mathrm{cm}^{3}$ are inherent to the quasistoichiometric $\mathrm{Ni}-\mathrm{Mn}-\mathrm{Ga}$ alloys $[8$,
$13,14,15]$. The spin-exchange parameter can be roughly estimated as $\alpha \approx T_{\mathrm{C}} l^{2} k_{\mathrm{B}}\left(\left|\mu_{\mathrm{B}}\right| M_{0}\right)^{-1}$, where $T_{\mathrm{C}}=360 \mathrm{~K}$ is the Curie temperature, $l=0.41 \times 10^{-8} \mathrm{~cm}$ is the distance between the magnetic atoms, $k_{\mathrm{B}}$ and $\mu_{\mathrm{B}}$ are the Boltzmann constant and the Bohr magneton, respectively.

The minimum conditions for the magnetic energy, Eq. (2), show that the magnetic field applied in the [101] direction stabilizes the state with $\mathbf{M} \|[101]$, when the inequality $H>H_{1} \equiv 2 K_{1} / M_{0}$ is fulfilled. For the abovementioned experimental values, the characteristic field value $H_{1}$ is of about 90 Oe.

The magnetoelastic resonance with the soft elastic mode takes place when $\rho \omega^{2} \approx C^{\prime} k^{2}$. In this case, Eq. (9) can be approximated by the simpler one,

$\left(\omega^{2}-\omega_{p h}^{2}\right)\left(\omega^{2}-\omega_{s w}^{2}\right)-9 \delta_{2}^{2} \gamma^{2} \rho^{-1} \omega_{m 1} k^{2}=0$, 
where $\omega_{s w}=\gamma M_{0}\left(\omega_{m 1} \omega_{m 2}\right)^{1 / 2}$. The solutions of the approximate equation,

$$
\omega_{ \pm}^{2}=\left[\omega_{p h}^{2}+\omega_{s w}^{2} \pm \sqrt{36 \delta_{2}^{2} \gamma^{2} k^{2} \rho^{-1} \omega_{m 1}+\left(\omega_{p h}^{2}-\omega_{s w}^{2}\right)^{2}}\right] / 2
$$

describe the dispersion of quasisound $\left(\omega_{-}\right)$and quasispin $\left(\omega_{+}\right)$waves in the crystal with soft shear modulus. The quasisound mode is gapless, while the spectrum of quasispin waves has a gap $\omega_{0}=\gamma\left(H-H_{1}\right)^{1 / 2}\left(H+H_{2}\right)^{1 / 2}$, where $H_{2} \equiv\left(K_{1}+K_{2} / 2\right) / M_{0} \approx 100$ Oe.

The quasisound and quasispin spectrum branches are shown in Figure. In view of the drastic difference in the shear moduli inherent to the different $\mathrm{Ni}-\mathrm{Mn}-\mathrm{Ga}$ alloys (the values $C_{\min }^{\prime} \sim 1 \mathrm{GPa}-60 \mathrm{GPa}$ were measured at the MT temperature for quasistoichiometric alloys), Figure, $a$ shows the spectra computed for three different values of shear elastic modulus; the field value $H=3.3 \mathrm{kOe}$ corresponds to the frequency $\omega_{0} / 2 \pi=9.1 \mathrm{GHz}$. The magnetoelastic interaction in the crystal with the shear modulus of $60 \mathrm{GPa}$ exhibits itself in the narrow interval of the wave vector values, which includes the resonance value $k_{0} \approx 2.4 \times 10^{5} \mathrm{~cm}^{-1}$. Far from the resonance, the magnetoelastic coupling do not change the dispersion curves of sound and spin waves noticeably. In the crystal with a shear modulus of $35 \mathrm{GPa}$, the interaction between the sound and spin waves results in the strong repulsion of the quasisound and quasispin spectrum branches and, hence, exhibits itself in a rather wide range of wave vector values $\Delta k \sim 10^{5} \mathrm{~cm}^{-1}$. (The "repulsion" is formally defined as $\Delta \omega \stackrel{\text { def }}{\equiv} \omega_{+}\left(k_{0}\right)-\omega_{-}\left(k_{0}\right)$, where $k_{0}$ satisfies the equation $\left.\omega_{s w}(k)=\omega_{p h}(k)\right)$. In the $\Delta k$ range, a noticeable nonlinearity of the dispersion of the quasisound branch can be observed. In the crystal with a shear modulus of $25 \mathrm{GPa}$, the magnetoelastic interaction does not change the dispersion of the elastic and spin waves noticeably. The shear moduli of a number of $\mathrm{Ni}-\mathrm{Mn}-\mathrm{Ga}$ alloys decrease from the values of few tens of gigapascals to the values of about one gigapascal, while the alloy temperature approaches the MT temperature. Therefore, the effect of the magnetoelastic interaction on the wave spectra should be most pronounced in a certain temperature interval above the MT temperature. In this interval, the value of shear elastic modulus must be close enough to $35 \mathrm{GPa}$.
1. A.I. Akhiezer, V.G. Baryakhtar, and S.V. Peletminsky, Zh. Eksp. Teor. Fiz., 35, 228 (1958).

2. C. Kittel, Phys. Rev. 110, 836 (1958).

3. M. Pomerantz, Phys. Rev. Lett. 7, 312 (1961).

4. A.I. Akhiezer, V.G. Baryakhtar, and S.V. Peletminsky, Spin Waves (North-Holland, Amsterdam, 1968).

5. V.G. Baryakhtar and E.A. Turov, in Spin waves and Magnetic Excitations, edited by A.S. Borovik-Romanov and S.K. Sinha (North-Holland, Amsterdam, 1988), Pt. 2, p. 333.

6. V.G. Baryakhtar, I.M. Vitebsky, Yu.G. Pashkevich, V.L. Sobolev, and V.V. Tarasenko, Zh. Eksp. Teor. Fiz. 87, 1028 (1984).

7. V.I. Ozhogin and V.L. Preobrazhenskii, J. Magn. Magn. Mater. 100, 544 (1991).

8. R.C. O'Handley and S.M. Allen, in Encyclopedia of Smart Materials, edited by M. Schwartz (Wiley, New York, 2002), p. 936.

9. P. Entel, V.D. Buchelnikov, M.E. Gruner, A. Hucht, V.V. Khovailo, S.K. Nayak, and A.T. Zayak, Sci. Forum 583, 21 (2008).

10. V.A. Chernenko and V.A. L'vov, Mater. Sci. Forum $\mathbf{5 8 3}$, 1 (2008).

11. V.V. Kokorin and M. Wuttig, J. Magn. Magn. Mater. 234, 25 (2001).

12. O. Heczko, J. Magn. Magn. Mater. 290, 846 (2005).

13. V.A. L'vov, I. Glavatskyy, and N. Glavatska, Mater. Sci. Forum 635, 131 (2010).

14. L. Dai, J. Cullen, and M. Wuttig, J. Appl. Phys. 95, 6957 (2004).

15. V.A. Chernenko and V.A. L'vov, Philos. Mag. 73, 999 (1996).

Received 01.08.11

\section{МАГНІТОПРУЖНИЙ РЕЗОНАНС У КРИСТАЛІ ПРИ ФАЗОВОМУ ПЕРЕХОДІ В ГРАТЦ}

\section{В.Г. Бар'яхтар, А.Г. Данилевич, В.А. Лъвов}

$\mathrm{P}$ е з ю м е

Передбачено можливість аномально сильного відштовхування гілок спектра хвиль квазізвука та спінових хвиль у кубічному феромагнітному кристалі завдяки фазовому переходу в гратці. 